\title{
Distribution and Density of Tsetse Flies (Glossinidae: Diptera) at the Game/People/Livestock Interface of the Nkhotakota Game Reserve Human Sleeping Sickness Focus in Malawi
}

\author{
Nkwachi Gondwe, ${ }^{1}$ Tanguy Marcotty, ${ }^{2}$ Sophie O. Vanwambeke, ${ }^{4}$ Claudia De Pus, ${ }^{2}$ \\ Misheck Mulumba, ${ }^{1}$ and Peter Van den Bossche ${ }^{2,3}$ \\ ${ }^{1}$ Centre for Ticks and Tick-Borne Diseases, Lilongwe, Malawi \\ ${ }^{2}$ Department of Animal Health, Institute of Tropical Medicine, Antwerp, Belgium \\ ${ }^{3}$ Department of Veterinary Tropical Diseases, University of Pretoria, Onderstepoort, South Africa \\ ${ }^{4}$ Department of Geography, Université catholique de Louvain, Louvain-la-Neuve, Belgium
}

\begin{abstract}
In large parts sub-Saharan Africa, tsetse flies, the vectors of African human or animal trypanosomiasis, are, or will in the foreseeable future, be confined to protected areas such as game or national parks. Challenge of people and livestock is likely to occur at the game/livestock/people interface of such infested areas. Since tsetse control in protected areas is difficult, management of trypanosomiasis in people and/or livestock requires a good understanding of tsetse population dynamics along such interfaces. The Nkhotakota Game Reserve, an important focus of human trypanosomiasis in Malawi, is a tsetse-infested protected area surrounded by a virtually tsetse-free zone. The abundance of tsetse (Glossina morsitans morsitans) along the interface, within and outside the game reserve, was monitored over 15 months using epsilon traps. A land cover map described the vegetation surrounding the traps. Few flies were captured outside the reserve. Inside, the abundance of tsetse at the interface was low but increased away from the boundary. This uneven distribution of tsetse inside the reserve is attributed to the uneven distribution of wildlife, the main host of tsetse, being concentrated deeper inside the reserve. Challenge of people and livestock at the interface is thus expected to be low, and cases of trypanosomiasis are likely due to people and/or livestock entering the reserve. Effective control of trypanosomiasis in people and livestock could be achieved by increasing the awareness among people of dangers associated with entering the reserve.
\end{abstract}

Key words: trypanosomiasis, tsetse, interface, control

\section{INTRODUCTION}

Tsetse-transmitted trypanosomiases are diseases of people and livestock that occur in a large part of sub-Saharan

Correspondence to: Peter Van denBossche, e-mail: pvdbossche@itg.be
Africa and are the result of a bite by an infected tsetse fly (Glossina spp.) (Maudlin et al., 2004). In southern Africa, the epidemiology of the disease varies from one locality to another and depends largely on the level of interaction between tsetse flies and their hosts. The nature of this interaction is subject to spatial and temporal variations. Within the tsetse-infested areas of southern Africa, four 
distinct epidemiological situations can be distinguished, i.e., (i) wildlife zones where people and livestock are absent, (ii) areas where people and livestock have been recently introduced into wildlife zones, (iii) areas where people and livestock reside in tsetse-infested zones and where large game animals are absent, and (iv) areas where people and livestock are present at the edge of wildlife zones (interfaces) (Van den Bossche, 2001). Especially game/people/ livestock interfaces are of particular importance and are likely to gain even more importance in the future. This is due to increasing population pressure and agricultural development, and the concomitant disappearance of suitable tsetse habitat outside protected areas such as national parks, game reserves, and forest reserves. As a result, tsetse flies will be more and more confined to these protected areas, with people and livestock being challenged by the tsetse flies at the edge of such protected areas. Such interfaces already exist in, for example, Malawi and part of the KwaZulu-Natal Province of South Africa (Van den Bossche et al., 2000, 2006). Although the eradication of tsetse in protected areas would be a permanent solution to the trypanosomiasis problem, environmental concerns often make tsetse control in such areas an unlikely option. Hence, other disease management strategies need to be developed to control human and/or livestock trypanosomiasis at game/livestock/people interfaces. Effective management requires a better understanding of the factors determining the contact between tsetse and susceptible people and livestock at the interface.

The Nkhotakota Game Reserve constitutes an important tsetse reservoir in the Central Region of Malawi. The game reserve is surrounded by cultivated areas with mixed livestock/agriculture, mainly subsistence, farming systems. The game reserve is an important focus of human sleeping sickness (Trypanosoma brucei rhodesiense) (Pugh, 1987). Moreover, livestock trypanosomiasis is considered to be a threat to livestock in the areas surrounding the game reserve (Van den Bossche et al., 2000; Van den Bossche, 2001). To improve the management of human and livestock trypanosomiasis along the Nkhotakota Game Reserve game/people/livestock interface, and to obtain a first insight into the dynamics and factors affecting the dynamics of the tsetse population at such an interface, a study was conducted to determine the distribution and density of tsetse along the edge and inside the game reserve.

\section{Materials And Methods}

\section{Study Area}

The Nkhotakota Game Reserve (NGR) is located in the Central Region of Malawi and has a surface of approximately 180,000 hectares. A buffer zone was established to demarcate the game reserve from the surrounding settled area, but in the absence of a physical barrier around the NGR, there has been extensive encroachment of people and their livestock into the buffer zone, and in some areas into the game reserve, resulting in the destruction of the natural vegetation (Fig. 1). The study was conducted in the Linga Extension Planning Area (EPA) situated between the Bua and Kaombe rivers East of the Nkhotakota Game Reserve. A total of 30,772 households live in the Linga EPA. It has a total livestock population of 1678 head of cattle, 12,042 goats, and 835 pigs (based on the March 2007 livestock census). Tsetse monitoring was restricted to Mphode Senior Traditional Authority of the Linga EPA.

The NGR is infested with Glossina morsitans morsitans and, to a much lesser extent, with G. brevipalpis (Van den Bossche et al., 2000). The vegetation in the NGR is mainly Miombo woodland, with Brachystegia and Jubernadia being the major tree species. An aerial survey conducted in 1997 reported wildlife such as elephants, buffalo, kudu, reedbuck, antelope, waterbuck, bushbuck, warthog, duiker, zebra, and baboons (JOFCA, 1997). The density of the wildlife in the reserve is generally low, and their distribution in the reserve varies (JOFCA, 1997).

The annual climatic cycle comprises three seasons: the warm, rainy season (from early November to late April); the cold, dry season (from early May to late August); and the hot, dry season (from early September to late October).

\section{Tsetse Surveillance Inside the Nkhotakota Game Reserve}

The tsetse population inside the NGR was monitored along two transects (Fig. 1) of epsilon traps (Hargrove and Langley, 1990) for a period of 15 consecutive months. A total of 50 traps were deployed at intervals of $250 \mathrm{~m}$ with the first transect (KKT) consisting of 31 traps, while the second transect (BCT) had 19 traps. Traps were deployed in open areas; trapping sites were geo-referenced using a global positioning system (GPS) and located on a land cover map derived from a SPOT-XS image dating from 


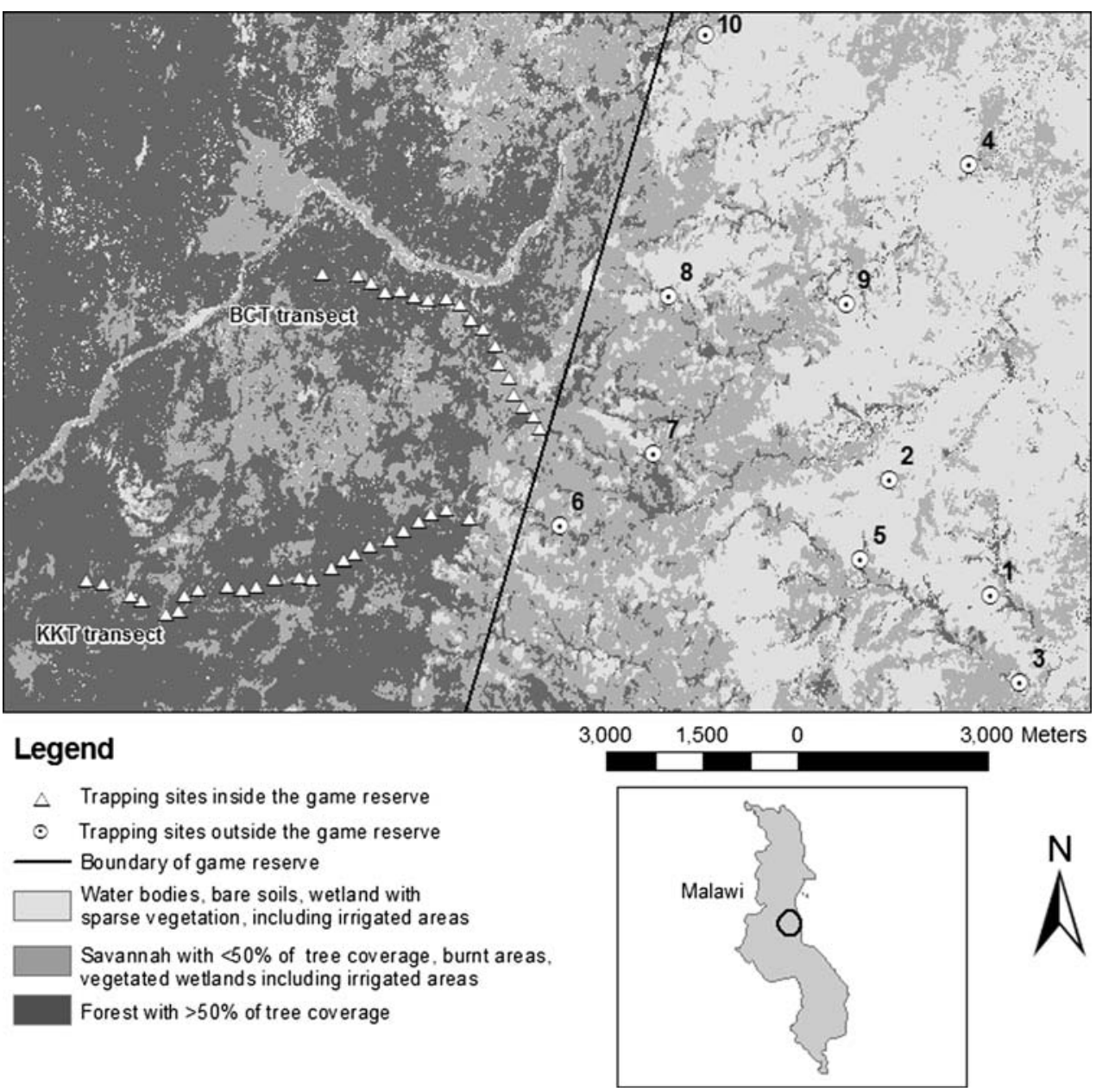

Figure 1. Location of traps along the two transects (BCT and KKT), both inside and outside the Nkhotakota Game Reserve, Malawi.
September 11, 2001 (Fig. 1). The image was geo-referenced using the 1/50,000 topographic map of Malawi. Maximum likelihood classification was then used to generate a land cover map at $20 \mathrm{~m}$ of resolution, with three vegetation classes representing habitats with varying suitability for $G$. $m$. morsitans. Vegetation class 1 represents woodland with trees covering more than $50 \%$ of the surface. Vegetation class 2 represents woodland with tree coverage of less than $50 \%$, burnt areas, and vegetated wetlands, including some irrigated fields. Finally, vegetation class 3 includes water bodies, bare soils wetlands with sparse vegetation, and some irrigated fields. The global accuracy for this map was $88 \%$. The distance between each of the traps and the Park's boundary was measured using the land cover map. For each of the traps, and in a buffer area of $200 \mathrm{~m}$ surrounding the trap, the proportion of each of the three vegetation classes was calculated.

Traps were baited with acetone released at approximately $200 \mathrm{mg} /$ hour. During the 15 -month observation period, traps were functional for $4-5$ consecutive days per month. During this period, harvesting of tsetse flies was conducted every morning. Records were kept of the number of tsetse (males and females) captured in each trap during each of the days of sampling. For each of the transects, a monthly mean index of apparent abundance (IAA) of tsetse was calculated as the number of flies (males and females) captured per trap, per trapping day.

Because the data were Poisson distributed, the number of tsetse flies recorded in each trap was analyzed in a crosssectional, Poisson regression in Stata software version 9.2 (StataCorp, 2006). The season was used as a discrete explanatory variable, whereas the distance from the Park boundary and the vegetation abundance (expressed as the proportion of vegetation type 1 in each buffer zone surrounding the trap) were used as continuous explanatory variables. The interactions between season and (1) distance to boundary, and (2) vegetation abundance, were tested. The likelihood ratio test was used to remove nonsignificant explanatory variables $(P>0.1)$. Finally, the different counts in individual traps were considered as repeated 
measures. The goodness-of-fit was tested in an ordinary Poisson regression (not cross-sectional), using the same explanatory variables, to confirm the validity of the model.

\section{Tsetse Survey Outside the NGR}

To determine if tsetse flies were present outside the NGR, two tsetse surveys were conducted. For this purpose, 10 sampling sites, with what appeared to be suitable tsetse habitat, were identified on the land cover map and located using a GPS. Traps were deployed at distances varying between $620 \mathrm{~m}$ and $8.2 \mathrm{~km}$ from the boundary of the game reserve (trapping sites 1-10 on Fig. 1).

A first survey was conducted for 10 consecutive days in the rainy season (December), and a second survey was conducted for 14 consecutive days in the dry season (July). During each of the seasons, four epsilon traps (baited as described above) were deployed at each site. Traps were visited at 2-3-day intervals, and records were kept of the number of tsetse flies captured in each of the traps.

\section{Results}

Due to access problems, traps were visited 13 times during the 15-month observation period. They were functional for a total of 55 trapping days.

\section{Tsetse Surveillance Inside and Along the Interface of the NGR}

During the observation period, a total of 757 G. m. morsitans (267 males and 490 females) was captured. The BCT transect accounted for 168 flies (38 males and 130 females), whereas a total of 589 flies (229 males and 360 females) were captured in traps of the KKT transect. The fly captures were not significantly affected by the season of capture $(P=0.27)$ (Fig. 2). However, the tsetse abundance along the transects increased with increasing distance inside the boundary of the NGR by a factor of 1.35 per $\mathrm{km}(95 \%$ confidence interval [CI]: 1.2-1.7) and by a factor of 1.02 with each percent increase in the proportion of vegetation belonging to vegetation class 1 (95\% CI: 1.0-1.04) (Fig. 3).

\section{Tsetse Survey Outside the Nkhotakota Game Reserve}

A total of five female G. m. morsitans was captured in traps located outside the NGR. Captures were restricted to the

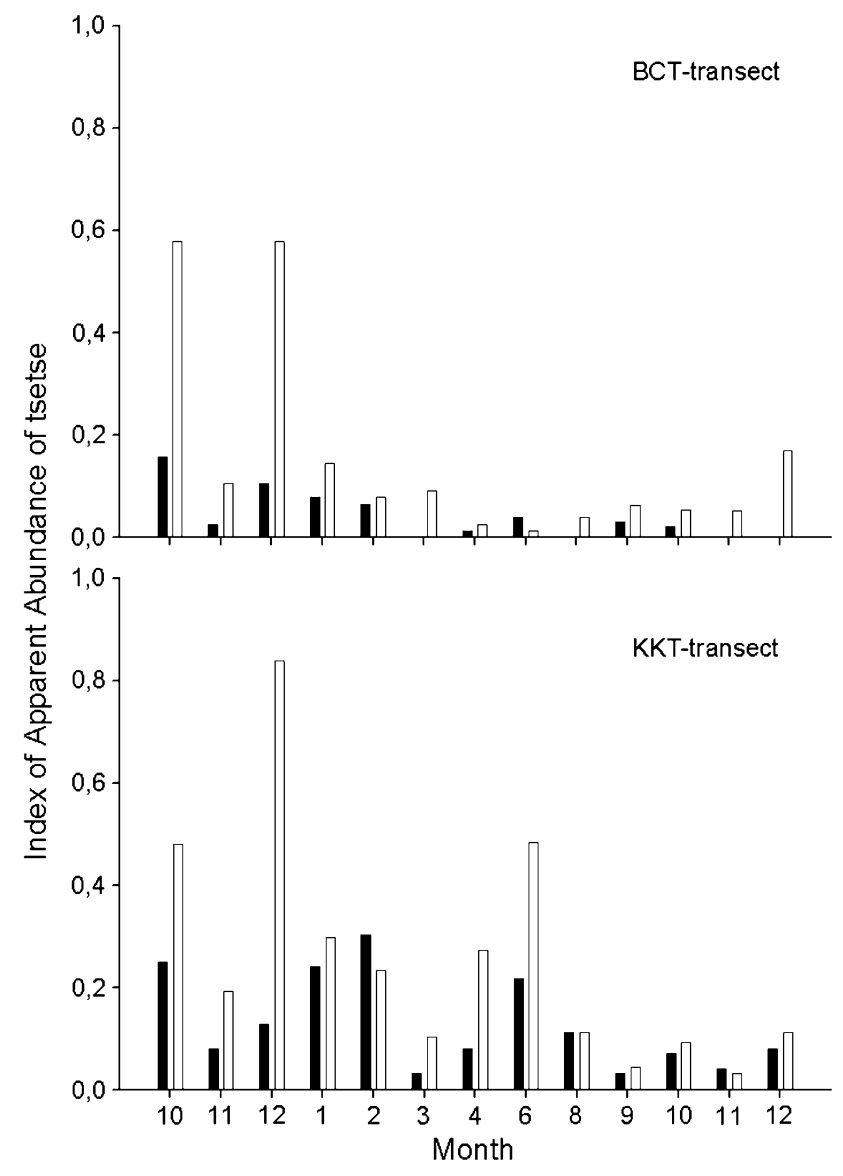

Figure 2. Monthly index of apparent abundance (IAA) of male (black bars) and female (white bars) Glossina morsitans morsitans captured along the BCT and KKT trap transects in the Nkhotakota Game Reserve, Malawi.

three sampling sites located closest to the NGR (Fig. 1). One tsetse fly was captured during the dry season survey (trapping site 7). The remaining four tsetse flies were captured during the rainy season (two flies at trapping site 7 , one fly at trapping site 6 , and one fly at trapping site 8 ).

\section{DISCUSSION}

The results of the longitudinal study show an uneven distribution of tsetse inside the NGR, and a low apparent abundance of tsetse flies outside the NGR. The differences in abundance inside and outside the NGR are attributed mainly to the human activity outside the game reserve and the concomitant destruction of the tsetse habitat in areas surrounding the NGR, making them less suitable for tsetse. The adverse effects of increasing human population on the density of tsetse flies and game animals have been known for a long time (Swynnerton, 1921), and were the main 


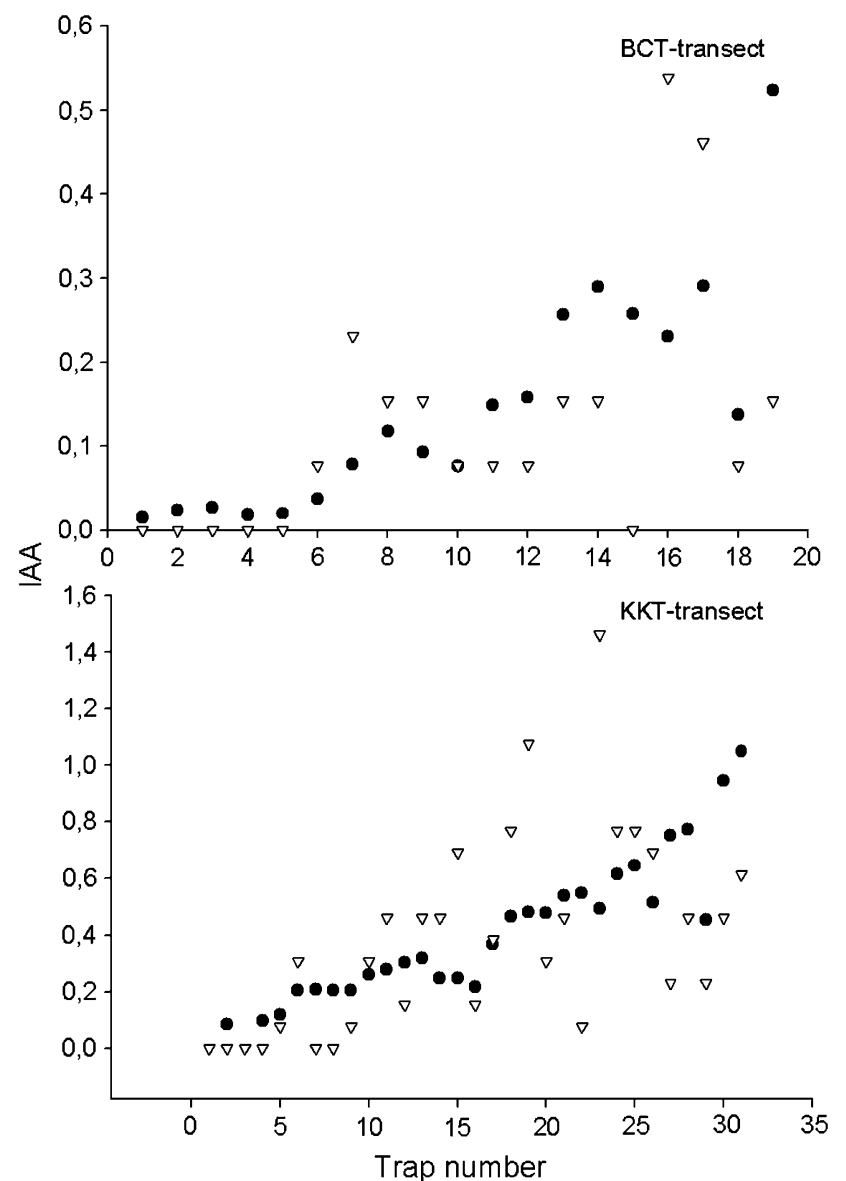

Figure 3. Observed $(\triangle)$ index of apparent abundance (IAA) of Glossina morsitans morsitans captured in individual traps along the BCT and KKT transects, and predicted ( $)$ IAA by the simplified cross-sectional Poisson model (using the distance to the Park and the vegetation index as explanatory variables).

reasons identified by Ford (1963) for the retreat of tsetse from extensive areas of Malawi (then Nyasaland). The subsequent concentration of tsetse flies in protected areas, such as national parks, game reserves, and forest reserves, where the natural tsetse habitat is still present, was confirmed during the tsetse and the trypanosomiasis surveys conducted under the auspices of the Regional Tsetse and Trypanosomiasis Control Project (Van den Bossche et al., 2000). The NGR is one of these remaining foci of tsetse flies in Malawi. However, tsetse density also seems to vary spatially within the focus. At the edge of the NGR, abundance is generally low. Further inside the NGR, on the other hand, the apparent abundance of tsetse increases and is similar to the apparent abundance of G. m. morsitans on the adjacent plateau areas of eastern Zambia (Van den Bossche and De Deken, 2002). According to the multivariate model, the apparent abundance of tsetse is not only significantly correlated with the density of the tree cover in the areas surrounding the traps, but also with the distance between the trapping site and the edge of the game reserve. It thus seems that factors other than vegetation affect the distribution of tsetse.

Although it is difficult to determine unequivocally what causes the observed differences in abundance inside the NGR, it is very likely due to the availability of hosts. For their survival, tsetse flies require regular bloodmeals. Other studies on G. m. morsitans in southern Africa have shown that this tsetse species is flexible with regard to host preference and can survive on wild and/or domestic hosts. Indeed, on the plateau area of eastern Zambia, G. m. morsitans depends almost entirely on cattle as the source of food, whereas in the Luangwa Valley of Zambia, game animals constitute the major source of food (Van den Bossche and Staak, 1997; Simukoko et al., 2007). It thus seems that the type of host is less important than the availability of hosts. On the plateau area of eastern Zambia, the availability of the host has been shown to affect the distribution of tsetse (Van den Bossche and De Deken, 2002). It is expected that the same applies to the distribution of tsetse inside the NGR. Since domestic animals are absent inside the NGR and their density in the areas surrounding the NGR is generally low, tsetse flies inside the game reserve will rely heavily on game animals as the source of food. Game animals in the NGR are not distributed equally (JOFCA, 1997). This is attributed to the high level of poaching, by people living in the surrounding villages, in the border areas of the NGR, and the subsequent concentration of game animals well inside the NGR. Hence, host availability for tsetse is expected to be much higher in the central areas of the game reserve, compared to the interfaces along which humans have settled. It is thus not surprising that tsetse flies are distributed accordingly with a much higher density inside the NGR, compared to the edge of the game reserve. This situation is aggravated by the establishment of human settlements in the buffer zones and the absence of fences preventing people from entering the game reserve. In this respect, the situation in the NGR seems to be completely different from the situation in the tsetse-infested Hluhluwe-iMmfolozi Park of the KwaZulu-Natal Province in South Africa. Here, the presence of fences and the control of poaching do result in the presence of game at the edges of the Park, and the presence of high-density populations of tsetse flies at the interface (Van den Bossche et al., 2006).

The NGR is one of the foci of human trypanosomiasis in Malawi. Based on the tsetse distribution and density 
data, and in accordance with data on the prevalence of livestock trypanosomiasis, challenge of humans at the interface or outside the NGR is expected to be low. Hence, challenge of people at the interface or outside the NGR is expected to contribute little to the overall prevalence of human sleeping sickness in the NGR human trypanosomiasis focus. It is much more likely that cases of human trypanosomiasis are the result of people penetrating several kilometers in the NGR.

The observed distribution of tsetse has important repercussions for the strategy to control trypanosomiasis along the NGR, and probably similar interfaces. Considering the observed situation, whereby the tsetse challenge at the edge and outside the NGR is low, due to deforestation, poaching, and absence of fences, it is questionable if the control of tsetse using stationary baits such as odor-baited insecticide-treated targets inside the NGR or along the interface of the NGR is necessary. Tsetse control would be a worthless exercise unless the goal were complete eradication from the isolated NGR focus. However, considering the difficulties associated with the control of tsetse flies, an equally effective way of controlling trypanosomiasis in people and livestock may be to create awareness among the people of the dangers associated with entering the game reserve, with or without livestock.

\section{ACKNOWLEDGMENTS}

This study would not have been successful without the support of individuals and institutions-too numerous to be mentioned. Thanks go to the Director and staff of the Department of Animal Health and Industry particularly those at Nkhotakota Rural Development Project for making available the resources needed to conduct the research. Special thanks go to Mr E. Maganga from the Department of Animal Health and Industry for his advice, efforts, and determination during the research. We thank Mr Kalingumbwa of the Nkhotakota Rural Development Project for his advice on the study area, and the farmersof the study area, facilitating the coordination of the research activities. This study was supported financially under the aegis of the Institutional Collaboration Project between the Belgian Development Cooperation and the Department of Veterinary Tropical Diseases of the University of Pretoria (South Africa).

\section{REFERENCES}

Ford J (1963) The distribution of vectors of African pathogenic trypanosomes. Bulletin of the World Health Organization 28:653-669

Hargrove JW, Langley PA (1990) Sterilizing tsetse (Diptera, Glossinidae) in the field-a successful trial. Bulletin of Entomological Research 80:397-403

CA JOF (1997) Final report of the master plan study on sustainable multiple-use resource management of Nkhotakota Wildlife Reserve, Malawi, Tokyo, Japan: Pasco International Inc.

Maudlin I, Holmes PH, Miles MA (2004) The trypanosomiases, CABI Publishing: Wallingford UK

Pugh RNH (1987) Resurgence of the tsetse threat in Malawi. Malawi Epidemiology Quarterly 1:6-10

Simukoko H, Marcotty T, Phiri I, Geysen D, Vercruysse J, Van den Bossche P (2007) The comparative role of cattle, goats and pigs in the epidemiology of livestock trypanosomiasis on the plateau of eastern Zambia. Veterinary Parasitology 147:231-238

StataCorp (2006) Stata Statistical Software: Release 9.2, College Station, TX: StataCorp

Swynnerton CFM (1921) An examination of the tsetse problem in North Mossurise, Portuguese East Africa. Bulletin of Entomological Research 11:315-385

Van den Bossche P (2001) Some general aspects of the distribution and epidemiology of bovine trypanosomosis in southern Africa. International Journal for Parasitology 3:592-598

Van den Bossche P, De Deken R (2002) Seasonal variations in the distribution and abundance of the tsetse fly, Glossina morsitans morsitans in eastern Zambia. Medical and Veterinary Entomology $16: 170-176$

Van den Bossche P, Staak C (1997) The importance of cattle as a food source for Glossina morsitans morsitans Westwood (Diptera: Glossinidae) in Katete District, Eastern Province, Zambia. Acta Tropica 5:105-109

Van den Bossche P, Esterhuizen J, Nkuna R, Matjila T, Penzhorn BL, Geerts S, et al. (2006) An update of the bovine trypanosomosis situation at the edge of the Hluhluwe-iMfolozi Park, Kwa-Zulu Natal Province, South Africa. Onderstepoort Journal of Veterinary Research 73:77-79

Van den Bossche P, Shumba W, Makhambera P (2000) The distribution and epidemiology of bovine trypanosomosis in Malawi. Veterinary Parasitology 88:163-176 